\title{
Enquête de 2016 auprès des lecteurs du Relevé des maladies transmissibles au Canada
}

\author{
P Huston ${ }^{1 *}$, NM Farrell², L Townley²
}

\section{Résumé}

Contexte : Le Relevé des maladies transmissibles au Canada (RMTC) est un journal scientifique revu par les pairs publié depuis 1975. En 2011, une enquête auprès des lecteurs a été réalisée afin d'orienter le processus de revitalisation du RMTC. À la fin de l'année 2016, cette enquête a été réitirée afin d'évaluer les progrès accomplis.

Objectif : Fournir des renseignements sur les résultats de l'enquête de 2016 auprès des lecteurs du RMTC qui a permis d'identifier le lectorat du RMTC et de recenser ses besoins, d'obtenir des commentaires sur le processus de revitalisation du journal et de chercher à obtenir d'autres suggestions d'amélioration.

Méthodologie : Une enquête auprès des lecteurs a été menée en ligne du 7 au 28 septembre 2016. Des invitations ont été envoyées par courriel aux abonnés du RMTC. Cette enquête s'est appuyée sur la version de 2011 et sa validité apparente a été vérifiée. L'analyse comportait des statistiques descriptives ainsi qu'une évaluation qualitative des commentaires pour les thèmes abordés.

Résultats : Au total, 549 personnes ont répondu à l'enquête (taux de participation de $12 \%$ ). Les répondants travaillaient en majorité dans le domaine de la santé publique (61\%), dans les soins cliniques (23\%), le milieu universitaire (16\%) et la médecine de laboratoire (9\%). Environ $45 \%$ des répondants recevaient le RMTC depuis moins de quatre ans, ce qui concorde avec le fait que le nombre d'abonnés a plus que doublé au cours de cette période. Plus de $90 \%$ des répondants ont indiqué qu'ils lisaient les articles publiés dans le RMTC (toujours : $15 \%$; souvent : $43 \%$; parfois : $35 \%$ ). Lorsqu'on leur demandait quelle était leur principale source d'information sur les maladies infectieuses au Canada, le RMTC était cité en premier par $72 \%$ des répondants. À la question " qu'est-ce qui vous plaît le plus en ce qui concerne le RMTC? ", les commentaires indiquaient généralement que ce journal fournissait un contenu canadien, qu'il était bien rédigé, qu'il était fondé sur des données probantes, et qu'il était intéressant et pertinent. La principale suggestion d'amélioration du RMTC consistait à demander son inscription à PubMed.

Conclusion : Les résultats de l'enquête indiquent que la revitalisation du RMTC a été une réussite et qu'elle répond à la nécessité pour le lectorat de disposer d'un journal scientifique sur les maladies infectieuses ayant un contenu canadien de haute qualité et pertinent. Conformément aux suggestions d'amélioration, le RMTC rejoindra la base de données PubMed au cours de l'année prochaine.

\section{Affiliations}

1 Bureau de la rédaction du RMTC, Direction générale de la prévention et du contrôle des maladies infectieuses, Agence de la santé publique du Canada, Ottawa (Ontario)

${ }^{2}$ Direction générale des communications et des affaires publiques, Santé Canada, Ottawa (Ontario)

${ }^{\star}$ Correspondance : patricia. huston@canada.ca

Citation proposée : Huston P, Farrell NM, Townley L. Enquête de 2016 auprès des lecteurs du Relevé des maladies transmissibles au Canada. Relevé des maladies transmissibles au Canada. 2017;43(2):61-4. https://doi.org/10.14745/ccdr.v43i02a04f

\section{Introduction}

Le Relevé des maladies transmissibles au Canada (RMTC) est un journal scientifique bilingue revu par les pairs et accessible en ligne qui est publié sans interruption depuis 1975. II a d'abord été publié par Santé Canada avant de l'être par l'Agence de la santé publique du Canada (ASPC). En 2011, après une réduction du calendrier de publication, une enquête auprès des lecteurs du RMTC a été menée afin d'orienter un possible renouvellement du journal. À cette époque, le RMTC comptait environ 2000 abonnés. Les lecteurs ont fait remarquer que le
RMTC devait se réinventer pour " publier de nouveau et en temps opportun des articles canadiens portant sur les maladies transmissibles ». Les répondants à l'enquête de 2011 ont indiqué qu'ils souhaitaient plus de rapports précoces d'éclosions accompagnés des possibilités de propagation (95\%), de rapports nationaux de surveillance sur les maladies à déclaration obligatoire (91\%) et d'articles de recherche sur les maladies infectieuses pertinents pour le Canada (91\%) [données non publiées]. 
Après l'enquête de 2011, le bureau de la rédaction a été rétabli et, en s'appuyant sur les résultats de l'enquête, la revitalisation du RMTC a été effectuée et le journal a commencé à publier des numéros réguliers avec des articles revus par les pairs en 2013. Aujourd'hui, son mandat consiste à publier rapidement des renseignements sur les maladies infectieuses actuelles et émergentes pertinentes au Canada afin d'orienter l'élaboration de politiques, de programmes et de pratiques. Le nombre d'abonnés a plus que doublé entre l'enquête de 2011 et celle de 2016. Le nombre d'abonnements mensuels est comptabilisé depuis 2014. Le nombre d'abonnements mensuels est comptabilisé depuis 2014. (Figure 1)

\section{Figure 1 : Abonnements au Relevé des maladies} transmissibles au Canada de janvier 2014 à août 2016

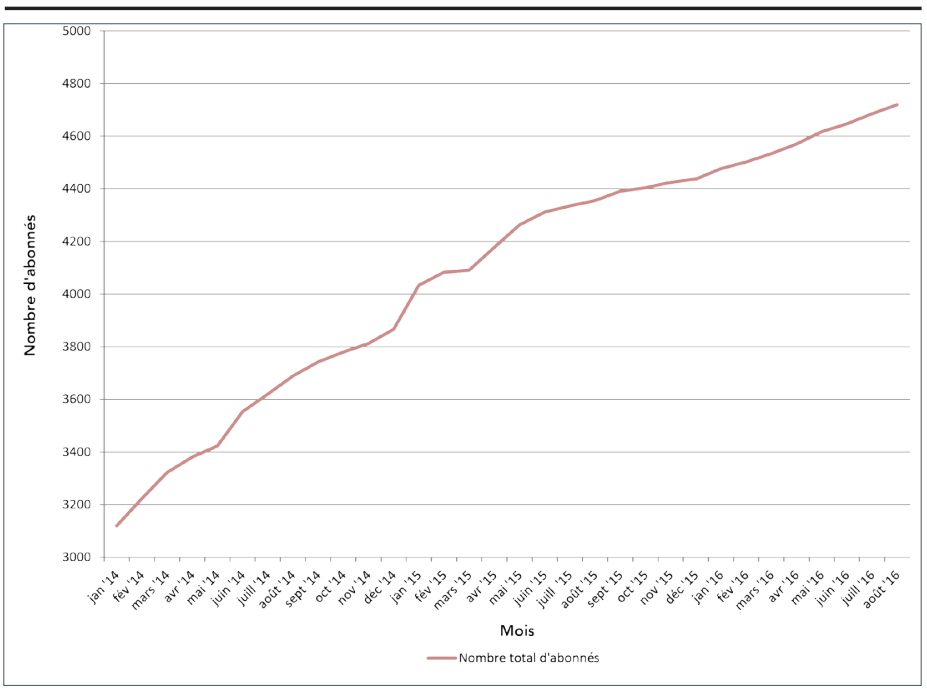

Au cours de l'été 2016 , le bureau de rédaction du RMTC a travaillé avec la Direction générale des communications et des affaires publiques de Santé Canada pour peaufiner et répéter l'enquête auprès des lecteurs. L'objectif de cette enquête de 2016 était d'identifier le lectorat du RMTC, d'obtenir des commentaires sur la période suivant la revitalisation du journal et de voir comment il serait possible de l'améliorer. Le présent document est un résumé du document Readership Survey Report (document non publié, Direction générale des communications et des affaires publiques, Santé Canada).

\section{Méthodologie}

Les méthodes d'enquête étaient fondées sur les normes relatives aux enquêtes en ligne du gouvernement fédéral (1). L'enquête en ligne de 2016 s'est appuyée sur l'enquête de 2011 et sa validité apparente a été vérifiée. Elle comprenait des questions portant sur les lecteurs, leur satisfaction à l'égard du journal, la manière dont ils reçoivent en général des renseignements sur les maladies infectieuses et la façon dont le RMTC pourrait être amélioré. La plupart des questions de l'enquête étaient à choix multiples et étaient suivies d'une section réservée aux commentaires qui comprenait une question sur la manière d'améliorer le RMTC. Après un essai pilote mené dans les deux langues officielles, une invitation à répondre à l'enquête en ligne a été envoyée par courriel aux abonnés du RMTC dont le nombre se chiffrait à 4716 .

L'enquête était accessible en ligne du 7 au 28 septembre 2016. Deux rappels ont été envoyés aux personnes n'ayant pas répondu à l'enquête afin d'optimiser le taux de participation. Le taux de participation a été calculé en divisant le nombre de répondants par la somme des courriels rejetés, des non-répondants et des répondants moins les adresses électroniques inexistantes. L'analyse des réponses comportait des statistiques descriptives ainsi qu'une évaluation qualitative des commentaires pour les thèmes abordés.

\section{Résultats}

\section{Lectorat du Relevé des maladies transmissibles au Canada et sources d'information}

Sur un total de 4716 abonnés, 17 adresses électroniques étaient inexistantes pour un total de 4699 participants. De ce nombre, 549 questionnaires ont été remplis pour un taux de participation de $12 \%$. En majorité, les répondants ont indiqué qu'ils travaillaient dans le domaine de la santé publique (61\%), dans les soins cliniques (23\%), le milieu universitaire (16\%) et la médecine de laboratoire (9\%). Ce total dépasse $100 \%$, car il était possible de choisir plus d'une réponse (par exemple, les soins cliniques ET le milieu universitaire). Un peu moins de $20 \%$ des répondants étaient âgés de 35 ans ou moins, $40 \%$ étaient âgés de 36 à 50 ans et $40 \%$ étaient âgés de plus de 50 ans. Près de $50 \%$ des répondants recevaient le RMTC depuis moins de quatre ans, ce qui concorde avec le fait que le nombre d'abonnés au RMTC a doublé au cours de cette période.

La plupart des lecteurs provenaient de I'Ontario et du Québec, même si l'on comptait des lecteurs de presque toutes les provinces et de tous les territoires, ainsi que d'autres provenant des États-Unis, de l'Union européenne et d'ailleurs (figure 2). Près de la moitié des répondants ont indiqué qu'ils avaient entendu parler du RMTC pour la première fois lors d'une conférence ou par l'entremise d'un collègue ou d'un enseignant.

\section{Figure 2 : Relevé des maladies transmissibles au Canada - Lectorat par emplacement}

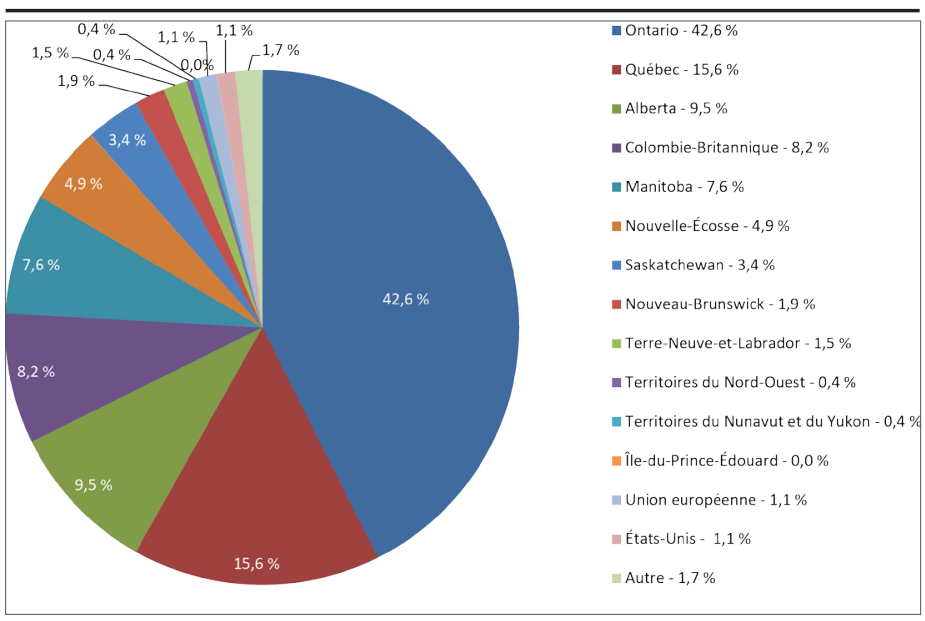

Les ressources utilisées le plus souvent par les répondants pour obtenir des renseignements sur les maladies infectieuses étaient les sites Web (75\%), les collègues (72\%) et les recherches documentaires en ligne (69\%). D'autres sources ont été signalées dans les commentaires, comme ProMED.

Lorsqu'on leur a demandé leurs principales sources de renseignements sur les maladies infectieuses et l'immunisation propres au Canada, la principale source était le RMTC (72 \%), suivie par les autorités locales de santé publique (52\%), les 
mises à jour des provinces ou des territoires (près de $50 \%$ ) et le Comité consultatif national de l'immunisation (CCNI) (50\%) (figure 3).

Figure 3 : Réponses à la question suivante de l'enquête de 2016 sur le Relevé des maladies transmissibles au Canada : "Quelles sont vos principales sources de renseignements sur les maladies infectieuses et I'immunisation propres au Canada (p. ex. éclosions locales, nouvelles recommandations vaccinales)? " ( $n=549$ )
RMTC

Autorité locale de santé publique

Comité consultatif national de l'immunisation (CCNI)

Mise à jour fournie par ma province ou mon territoire

Guide canadien d'immunisation

Canada.ca/santé

Médias

Experts locaux

Autre

Aucune réponse

\section{$72.3 \%$}

$52.1 \%$

$49.5 \%$

$49.4 \%$

$45.0 \%$

$31.1 \%$

$27.1 \%$

$20.0 \%$

$18.8 \%$ $2.0 \%$

\section{Commentaires sur le Relevé des maladies transmissibles au Canada revitalisé}

Plus de $90 \%$ des répondants ont déclaré lire les articles du RMTC (toujours : $15 \%$; souvent : $43 \%$; parfois : $35 \%$ ) et estimaient que les types $d$ 'articles suivants étaient utiles ou très utiles :

- Communications rapides sur les éclosions qui peuvent se propager $(90 \%)$

- Lignes directrices sur les maladies transmissibles de I'ASPC ou du CCNI (87 \%)

- Articles de recherche sur les maladies infectieuses pertinents pour le Canada (86\%)

- Rapports nationaux de surveillance sur les maladies à déclaration obligatoire (84\%)

Des commentaires ont été fournis par $62 \%$ des répondants $(n=340)$ dans la section fournie à cet effet. Les points forts du RMTC les plus souvent mentionnés étaient son contenu canadien, sa haute qualité et sa pertinence. À la question "qu'est-ce qui vous plaît le plus en ce qui concerne le RMTC?", les répondants formulaient typiquement les commentaires suivants : " canadien, bien rédigé, fondé sur des données probantes »; " articles intéressants »; "d'actualité »; " pertinent pour mon travail $»$; « concis et fiable ».

\section{Suggestions d'amélioration et prochaines étapes}

À la question «selon vous, quelle est la meilleure façon d'améliorer le RMTC? ", près de $50 \%$ des répondants ont formulé un commentaire $(n=266)$. Les commentaires les plus fréquents étaient les suivants : " continuez votre excellent travail » et "faites en sorte que le journal soit répertorié sur PubMed/MEDLINE ». Les autres suggestions étaient les suivantes:

- Sensibilisez les étudiants dans les universités et donnez des présentations

- Intégrez plus de rapports sur les éclosions d'origine alimentaire
- Ayez plus d'articles rédigés par des auteurs de tout le Canada

- Publiez le plus rapidement possible les données de surveillance et les rapports d'éclosion, notamment

Lorsqu'on leur a posé la question, $40 \%$ des répondants ont indiqué qu'ils aimeraient pouvoir accéder au RMTC à l'aide d'une application pour téléphones intelligents ou tablettes. Cependant, si ladite application comprenait le RMTC et d'autres ressources, comme le Guide canadien d'immunisation, les Lignes directrices canadiennes sur les infections transmissibles sexuellement et les Normes canadiennes pour la lutte antituberculeuse, $46 \%$ des répondants ont indiqué qu'ils la téléchargeraient. Les répondants indiquaient typiquement le commentaire suivant : " j'aimerais être en mesure d'accéder au RMTC à partir de mon iPhone ».

\section{Discussion}

Les lecteurs du RMTC proviennent de tout le Canada et du monde entier. La plupart des répondants travaillent dans les domaines de la santé publique et des soins cliniques. Les lecteurs apprécient le contenu revitalisé du RMTC, sa haute qualité ainsi que sa pertinence, et citent le journal comme leur principale source de renseignements sur les maladies infectieuses propres au Canada. En 2011, les lecteurs du RMTC avaient demandé des communications plus rapides, des résumés des recommandations du Comité consultatif et des articles de recherche sur les maladies infectieuses. Ces types d'articles ont été publiés dans le RMTC au cours des trois dernières années, et 85 à $90 \%$ des lecteurs les ont trouvés utiles.

Depuis la revitalisation du RMTC, son taux d'abonnement a plus que doublé. Les répondants à l'enquête ont proposé plus d'activités de sensibilisation, des auteurs canadiens, une plus grande rapidité et une application mobile. Toutefois, la suggestion d'amélioration la plus fréquente concernait l'intégration du journal au répertoire de PubMed.

La principale limite de ce sondage était son taux de réponse, lequel se chiffrait à $12 \%$. Bien que cela ne soit pas inhabituel pour un sondage en ligne, il n'est pas certain que les répondants soient représentatifs de l'ensemble du lectorat du RMTC.

L'enquête auprès des lecteurs confirme que le RMTC a fait des progrès importants au cours des cinq dernières années et qu'il représente pour ses lecteurs la source incontournable pour obtenir des renseignements pratiques et faisant autorité sur les maladies infectieuses au Canada. Nous avons le plaisir de vous annoncer que le RMTC a satisfait aux exigences de PubMed en matière de contenu scientifique et que l'intégralité des articles sera disponible dans PubMed en 2017.

\section{Collaborateurs}

Un grand merci aux personnes suivantes qui ont participé à l'élaboration de l'enquête et du présent article :

- Lidiya Tsegaye a rédigé des ébauches pour le questionnaire d'enquête

- Kyla Tyson a présenté les données reliées à l'abonnement, ella a mené l'analyse des commentaires figurant dans les réponses à l'enquête et a rédigé la première ébauche de l'article

- Mylène Poulin a fourni des commentaires sur les ébauches initiales du questionnaire d'enquête et les ébauches de l'article 


\section{Remerciements}

Un grand merci à tous les collaborateurs du RMTC ainsi qu'à toutes les personnes ayant répondu à l'enquête du RMTC.

\section{Conflit d'intérêts}

La $D^{\text {re }}$ Patricia Huston est la rédactrice scientifique en chef du RMTC et s'est récusée des décisions éditoriales relatives au présent article. La rédactrice associée, Dre Hilary Robinson, a pris les décisions éditoriales relatives au présent article.

\section{Financement}

L'ASPC soutient financièrement le RMTC.

\section{Référence}

1. Services publics et Approvisionnement Canada. Normes pour la recherche sur l'opinion publique effectuée par le gouvernement du Canada - Sondages en ligne [Internet]. Ottawa (ON): Gouvernement du Canada; [mise à jour le 19 nov 2014; consulté le 10 jan 2017]. Disponible à l'adresse : https://www.tpsgc-pwgsc.gc.ca/rop-por/enligne-online-fra. html\#s8.

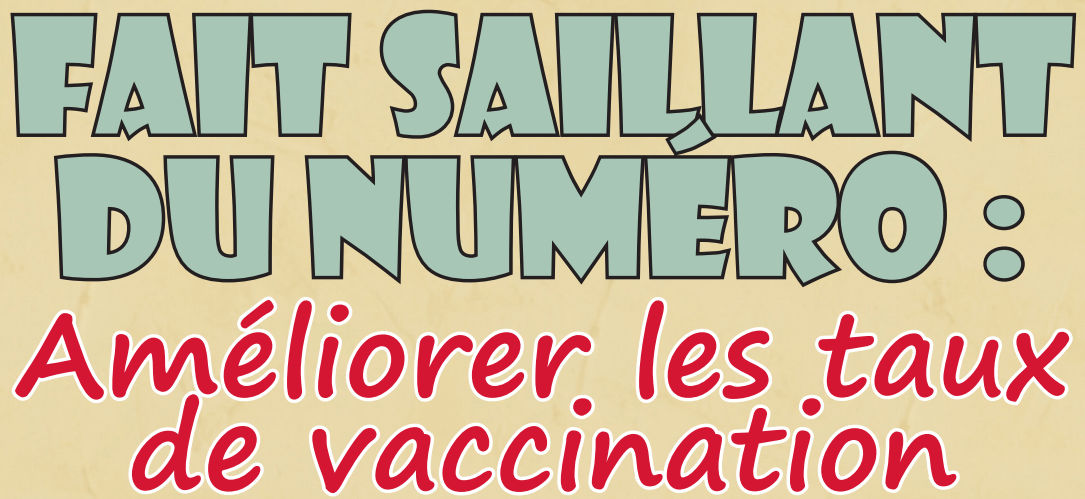

Dif

- Comment améliorer les taux de vaccination

- Comment aborder l'hésitation face aux vaccins

LIRE LE NUMÉRO:
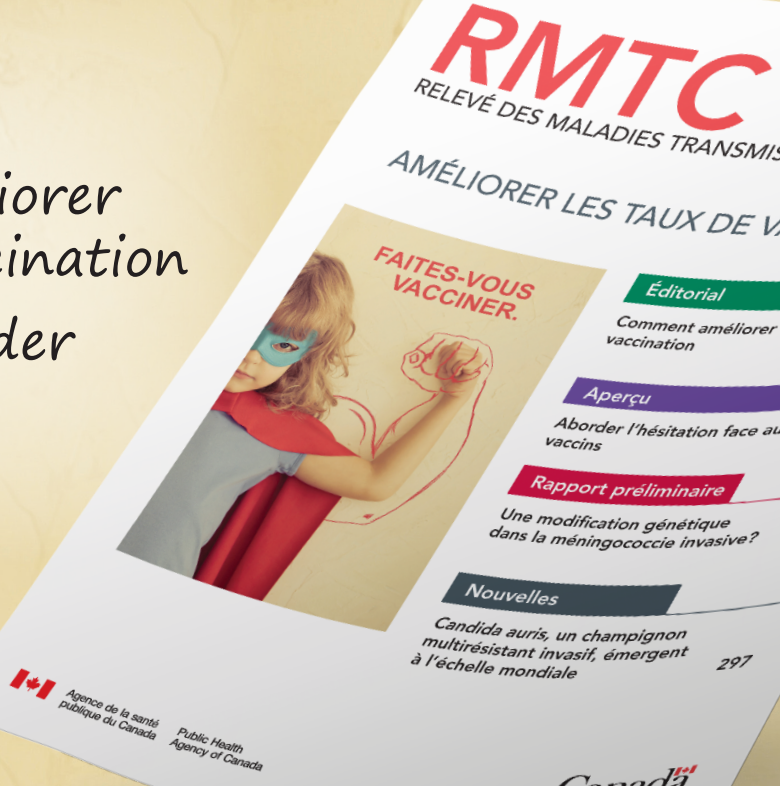

AMEOLIRANSMISSIBLES AU CANADA

AMELIORER LES TAUX DE VACCINATION

Recherche Web

RMTC+taux de vaccination a

